\title{
Training program for visually impaired and blind people on cardiopulmonary resuscitation and use of semi-automatic defibrillator
}

Graciotti P. 1., Messi D. 2 ,Virgini G. 3, Gabbanelli M. 3 , Mariotti V. 4

ASUR Marche - Area Vasta 2, Ancona, Italy, 2 AOU Ospedali Riuniti, Ancona, Italy, 3 INRCA, Ancona, Italy, 4 Opere Laiche Lauretane e Hermes, Loreto, Italy

\section{Introduction}

Cardiac arrest affects over 60,000 Italians a year.

The timely intervention of a bystander is essential to save a life.

In this scenario, the theme of the project is inserted, that is born from the request of the Italian Union of Blind and Visually Impaired Ancona (UICAM) to instruct the members on resuscitation techniques.

A study, experimentation and revision of the standard BLSD of IRC procedures were needed to make them accessible to visually impaired people. experience, encouraging mobility and orientation, and proposing the new instrument according to the following steps: motivation, perceptual knowledge, use of the instrument, progressively reducing the aid.
The instructor plays the role of guide of

\section{Materials and Methods}

The entire experimental project was conducted partially sighted, 3 sighted people.

The main changes made to the standard sequence involve the aid of a bystander for the evaluation of initial safety and during the use of the defibrillator, the use of a hand in place of sight to evaluate the thoracic excursion during the breath, and finally the necessity of specify the visual disability to the central operator.

Preliminary meeting with psychologist, documents data collection, satisfaction questionnaires, checklist for participant evaluation, BLSD manual for blind and visually impaired in Braille were developed.

\begin{tabular}{|c|c|c|c|c|c|c|}
\hline & Steps & $\begin{array}{l}\text { December } \\
2016\end{array}$ & $\begin{array}{l}\text { January } \\
2017\end{array}$ & \begin{tabular}{|l|} 
February \\
2017
\end{tabular} & $\begin{array}{l}\text { March } \\
2017\end{array}$ & \begin{tabular}{|l} 
April \\
2017
\end{tabular} \\
\hline 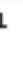 & $\begin{array}{l}\text { Bibliographic } \\
\text { research }\end{array}$ & $\mathrm{x}$ & $x$ & & & \\
\hline & Content processing & & & $x$ & & \\
\hline & \begin{tabular}{|l|}
$\begin{array}{l}\text { Experimentation and } \\
\text { comparison with a } \\
\text { psychologist }\end{array}$ \\
\end{tabular} & & & $x$ & & \\
\hline & \begin{tabular}{|l|} 
Modification of the \\
procedures
\end{tabular} & & & $\mathrm{x}$ & $\mathrm{x}$ & \\
\hline 5 & Training meeting & & & & x & \\
\hline 5 & Data processing & & & & $x$ & $x$ \\
\hline & $\begin{array}{l}\begin{array}{l}\text { Presentation of } \\
\text { results }\end{array} \\
\end{array}$ & & & & & $x$ \\
\hline \multicolumn{7}{|c|}{ Table 1. Timing } \\
\hline
\end{tabular}
on a group of 13 people, including 7 blind, 3

Results

The theoretical-practical training meeting took place at the UICAN headquarters, where the participants were successfully trained in resuscitation techniques.

In fact, $100 \%$ of the candidates passed the test, of which $62 \%$ without errors.

The remaining $38 \%$ experienced difficulties mainly with the use of the defibrillator and plates application about time factor

Mean satisfaction 4,8 (scale 1-5).

\begin{tabular}{|c|c|c|c|c|}
\hline Questions & Graduation & Average & Trend & Median \\
\hline $\begin{array}{l}\text { Performer } \\
\text { manual }\end{array}$ & $1-10$ & 8,9 & 8 & 9 \\
\hline Contents & $1-10$ & 9,7 & 10 & 10 \\
\hline $\begin{array}{l}\text { Presentation } \\
\text { course }\end{array}$ & $1-5$ & 4,5 & 3 & 5 \\
\hline Aims & $1-5$ & 5 & 5 & 5 \\
\hline $\begin{array}{l}\text { Presentation } \\
\text { and technique } \\
\text { AED }\end{array}$ & $1-5$ & 4,7 & 4 & 5 \\
\hline $\begin{array}{l}\text { Algorithm and } \\
\text { security }\end{array}$ & $1-5$ & 4,8 & 4 & 5 \\
\hline $\begin{array}{l}\text { Obviousness } \\
\text { sequenze } \\
\text { presentation }\end{array}$ & $1-5$ & 5 & 5 & 5 \\
\hline $\begin{array}{l}\text { Safety } \\
\text { assessment }\end{array}$ & $1-5$ & 4,8 & 4 & 5 \\
\hline Open airways & $1-5$ & 5 & 5 & 5 \\
\hline Breathing & $1-5$ & 4,8 & 4 & 5 \\
\hline $\begin{array}{l}\text { Chest } \\
\text { compressions }\end{array}$ & $1-5$ & 5 & 5 & 5 \\
\hline Activation AED & $1-5$ & 4,8 & 4 & 5 \\
\hline
\end{tabular}

Bigham B.L., Koprowicz K, Aufderheide T.P., (2010 Prehospital Emergency Care 14: 355-360 Ciaramell B. (2011) , Soccorso di base, Nozioni o tecniche. Piccin Editore

Chiaranda M. (2015) Urgenze ed emergenze, III Edizione, Piccin Editore

Del Rossi G., Dubose D, Scott N. (2014), Prehospital Emergency Care, 18: 539-543

Harrison P.R, Timmons S. (2006) Resuscitation, 71: 80-8: Nettina S.M.(2008) II Manuale dell'infermiere, II edizione, Piccin Editore

Perkins G.D., Travers A.H., Considine J. (2015), Adult Basic Life Support and Automated External Defibrillation. 95: 81-98

\begin{tabular}{|c|c|c|}
\hline Performance & Standard procedure & Modified procedure \\
\hline Scene safety assessment & Independent & Bystander employee \\
\hline Breathing assessment & $\begin{array}{l}\text {-See chest excursion-Listen to } \\
\text { breathing noises -Sense air in the } \\
\text { face - GAS in Italian }\end{array}$ & $\begin{array}{l}- \text { - Hand on the chest } \\
\text {-Listen to breathing noises } \\
\text {-Sense air in the face } \\
\text { MAS in tralian }\end{array}$ \\
\hline Emergency alert & $\begin{array}{l}\text { "Help, unconscious and does not } \\
\text { breathe person" }\end{array}$ & $\begin{array}{l}\text { + "I am a blind person but } \\
\text { can help" }\end{array}$ \\
\hline Safety during AED use & Indipendent & Bystander employee \\
\hline
\end{tabular}

\section{Conclusions} person has the skills necessary to act in emergency situations, so he is able to save a life before help arrives.
The project has shown that a blind

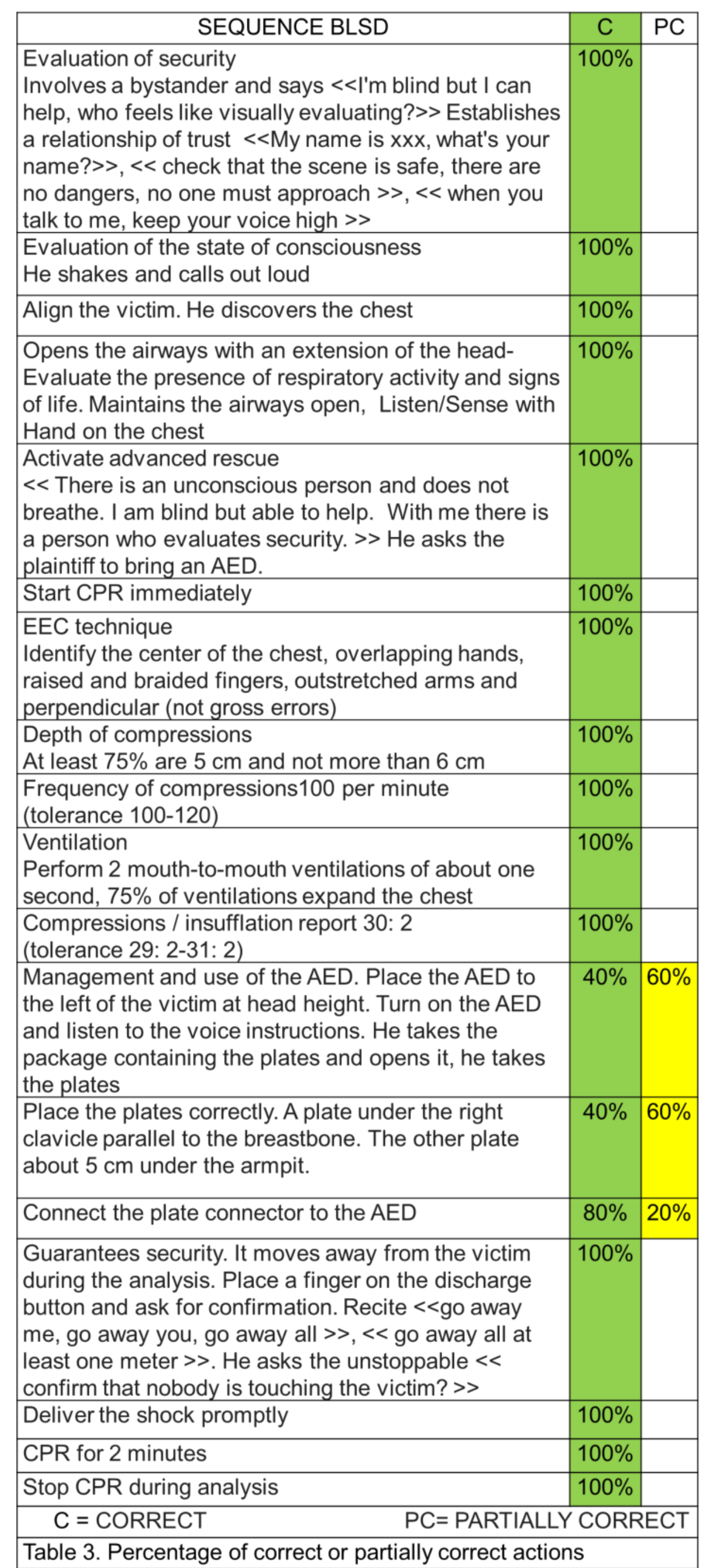

